\title{
Recovery of signals under the condition on RIC and ROC via prior support information
}

\author{
Wengu Chen ${ }^{1}$, Yaling $\mathrm{Li}^{2}$ \\ ${ }^{1}$ Institute of Applied Physics and Computational Mathematics \\ Beijing, 100088, China \\ ${ }^{2}$ Graduate School, China Academy of Engineering Physics \\ Beijing, 100088, China \\ Email: chenwg@iapcm.ac.cn, leeyaling@126.com
}

April 29, 2018

\begin{abstract}
In this paper, the sufficient condition in terms of the RIC and ROC for the stable and robust recovery of signals in both noiseless and noisy settings was established via weighted $l_{1}$ minimization when there is partial prior information on support of signals. An improved performance guarantee has been derived. We can obtain a less restricted sufficient condition for signal reconstruction and a tighter recovery error bound under some conditions via weighted $l_{1}$ minimization. When prior support estimate is at least $50 \%$ accurate, the sufficient condition is weaker than the analogous condition by standard $l_{1}$ minimization method, meanwhile the reconstruction error upper bound is provably to be smaller under additional conditions. Furthermore, the sufficient condition is also proved sharp.
\end{abstract}

Keywords Compressed sensing, Restricted isometry property, Restricted orthogonality constant, Weighted $l_{1}$ minimization, Sparse signal recovery

Mathematics Subject Classification (2010) 94A12, 90C59, 94A15

\section{Introduction}

Compressed sensing shows that it is highly possible to reconstruct sparse signals from what was previously believed to be incomplete information 10, 13]. The fundamental goal in compressed sensing is to recover a high dimensional sparse signal based on a small number of linear measurements, possibly corrupted by noise. This can be compactly described via

$$
y=A x+z,
$$


where $A$ is a given $n \times N$ sensing matrix with $n \ll N$, i.e., using very few measurements, $y \in \mathbb{R}^{n}$ is a vector of measurements, and $z \in \mathbb{R}^{n}$ is the measurement error ( $z=0$ means no noise). One needs to reconstruct the unknown signal $x \in \mathbb{R}^{N}$ based on $A$ and $y$. In general, the solutions to the underdetermined systems of linear equations (1.1) are not unique. In order to recover $x$ uniquely, additional assumptions on $A$ such as restricted isometry property and $x$ such as sparsity are needed. A vector $x \in \mathbb{R}^{N}$ is $k$-sparse if $\|x\|_{0}=|\operatorname{supp}(x)| \leq k$, where $\operatorname{supp}(x)=\left\{i: x_{i} \neq 0\right\}$ is the support of $x$. Then the most natural approach for solving this problem is to find the sparsest solution in the feasible set of possible solutions. In the noiseless case, it can be cast as the $l_{0}$ minimization problem as below [10, 13, 20, 26]:

$$
\underset{x \in \mathbb{R}^{N}}{\operatorname{minimize}}\|x\|_{0} \quad \text { subject to } A x=y .
$$

It was proved that when measurements $n>2 k$ and $A$ is in general position (any collection of $n$ columns of $A$ is linearly independent), then any $k$-sparse signals can be exactly recovered [14]. However, $l_{0}$ minimization problem is a combinatorial problem which becomes intractable in the high dimensional settings. Hence, solving it directly is NP-hard.

Candès and Tao [12] then proposed the following constrained $l_{1}$ minimization method:

$$
\underset{x \in \mathbb{R}^{N}}{\operatorname{minimize}} \quad\|x\|_{1} \quad \text { subject } \quad \text { to } \quad\|y-A x\|_{2} \leq \epsilon .
$$

It can be viewed as a convex relaxation of $l_{0}$ minimization. To recover sparse signals via constrained $l_{1}$ minimization, Candès and Tao [12] also introduced the notion of Restricted Isometry Property (RIP), which is one of the most commonly used frameworks for compressive sensing. The definition of RIP is as follows.

Definition 1.1. Let $A \in \mathbb{R}^{n \times N}$ be a matrix and $1 \leq k \leq N$ is an integer. The restricted isometry constant $(R I C) \delta_{k}$ of order $k$ is defined as the smallest nonnegative constant that satisfies

$$
\left(1-\delta_{k}\right)\|x\|_{2}^{2} \leq\|A x\|_{2}^{2} \leq\left(1+\delta_{k}\right)\|x\|_{2}^{2},
$$

for all $k$-sparse vectors $x \in \mathbb{R}^{N}$. Note that for $k_{1} \leq k_{2}, \delta_{k_{1}} \leq \delta_{k_{2}}$.

Thus, $l_{1}$ minimization has been proved an effective way to recover sparse signals in many settings [2, 3, 5 9 9, 12, 23]. Candès, Romberg and Tao first gained the sufficient condition for stable recovery by $l_{1}$ minimization method [9]. In [6], Cai and Zhang applied the following $l_{1}$ minimization

$$
\underset{x \in \mathbb{R}^{N}}{\operatorname{minimize}}\|x\|_{1} \quad \text { subject to }\|y-A x\|_{2} \in \mathcal{B},
$$

where $\mathcal{B}$ is a bounded set determined by the noise structure. In particular, $\mathcal{B}$ is taken to be $\{0\}$ in the noiseless case. Here they considered the following $l_{2}$ bounded noise and Dantzing Selector noise settings

$$
\mathcal{B}^{l_{2}}(\varepsilon)=\left\{z:\|z\|_{2} \leq \varepsilon\right\}
$$


and

$$
\mathcal{B}^{D S}(\varepsilon)=\left\{z:\left\|A^{T} z\right\|_{\infty} \leq \varepsilon\right\}
$$

Cai and Zhang [6] provided a sharp sufficient condition $\delta_{t k}<\sqrt{\frac{t-1}{t}}$ with $t \geq 4 / 3$ which can guarantee the exact recovery of all $k$-sparse signals in the noiseless case and stable recovery of approximately sparse signals in the noise case by $l_{1}$ minimization method (1.4) with (1.5) and (1.6). 7].

In addition, the restricted orthogonality constant is also important in compressed sensing [2, 3,

Definition 1.2. Let $A \in \mathbb{R}^{n \times N}$ be a matrix and $1 \leq k_{1}, k_{2} \leq N$ be integers with $k_{1}+k_{2} \leq N$, the restricted orthogonality constant $(R O C) \theta_{k_{1}, k_{2}}$ of order $\left(k_{1}, k_{2}\right)$ is defined as the smallest nonnegative constant that satisfies

$$
|\langle A u, A v\rangle| \leq \theta_{k_{1}, k_{2}}\|u\|_{2}\|v\|_{2},
$$

for all $k_{1}$-sparse vectors $u \in \mathbb{R}^{N}$ and $k_{2}$-sparse vectors $v \in \mathbb{R}^{N}$ with disjoint supports. Note that for $k_{1} \leq k_{2}$ and $k_{1}^{\prime} \leq k_{2}^{\prime}, \theta_{k_{1}, k_{1}^{\prime}} \leq \theta_{k_{2}, k_{2}^{\prime}}$.

It also has been shown that $l_{1}$ minimization can recover a sparse signal under various conditions on $\delta_{k}$ and $\theta_{k_{1}, k_{2}}[2] 5,7,11,12,15$, 17]. For example, $\delta_{k}+\theta_{k, k}+\theta_{k, 2 k}<1[12], \delta_{2 k}+\theta_{k, 2 k}<1$ [11], $\delta_{1.5 k}+\theta_{k, 1.5 k}<1$ [5] and $\delta_{1.25 k}+\theta_{k, 1.25 k}<1$ [2]. Cai and Zhang [7] also established a sharp sufficient condition in terms of RIC and ROC to achieve the stable and robust recovery of signals in both noiseless and noisy cases via $l_{1}$ minimization method. In fact, Cai and Zhang [7] proved that $\delta_{a}+C_{a, b, k} \theta_{a, b}<1$ can ensure stable and robust recovery of signals via $l_{1}$ minimization method (1.4) with (1.5) and (1.6). Moreover, for any $\varepsilon>0, \delta_{a}+C_{a, b, k} \theta_{a, b}<1+\varepsilon$ is not sufficient to guarantee the exact and stable recovery of all $k$-sparse signals via any methods.

It is worthy of noting that compressed sensing is a nonadaptive data acquisition technique since $A$ is independent of $x$, the signal being measured. The $l_{1}$ minimization method (1.3) is also itself nonadaptive as a result of no prior information on the signal $x$ being used in (1.4). In practical examples, however, the estimate of the support of the signal or of its largest coefficients may be possible to be drawn. Incorporating prior information is very useful for recovering signals from compressive measurements. Thus, the following weighted $l_{1}$ minimization method which incorporates partial support information of the signals has been introduced to replace standard $l_{1}$ minimization

$$
\underset{x \in \mathbb{R}^{N}}{\operatorname{minimize}}\|x\|_{1, \mathrm{w}} \quad \text { subject } \quad \text { to } \quad\|y-A x\|_{2} \leq \epsilon,
$$

where $\mathrm{w} \in[0,1]^{N}$ and $\|x\|_{1, \mathrm{w}}=\sum_{i} \mathrm{w}_{i}\left|x_{i}\right|$. Reconstructing compressively sampled signals with partially known support has been previously studied in the literature; see [1, 16, 18, 19, 21, 22, 24]. 
Borries, Miosso and Potes in [1], Khajehnejad et al. in [19], and Vaswani and Lu in [24] introduced the problem of signal recovery with partially known support independently. The works by Borries et al. in [1], Vaswani and $\mathrm{Lu}$ in [21, 24, 25] and Jacques in [18] incorporated known support information using weighted $l_{1}$ minimization approach with zero weights on the known support, namely, given a support estimate $\widetilde{T} \subset\{1,2, \ldots, N\}$ of unknown signal $x$, setting $\mathrm{w}_{i}=0$ whenever $i \in \widetilde{T}$ and $\mathrm{w}_{i}=1$ otherwise, and derived sufficient recovery conditions. Friedlander et al. in [16] extended weighted $l_{1}$ minimization approach to nonzero weights. They allow the weights $\mathrm{w}_{i}=\omega \in[0,1]$ if $i \in \widetilde{T}$. Since Friedlander et al. incorporated the prior support information and consider the accuracy of the support estimate, they derived the stable and robust recovery guarantees for weighted $l_{1}$ minimization which generalize the results of Candès, Romberg and Tao in [9]. They actually improved the recovery guarantees of $l_{1}$ minimization problem (1.3) by using weighted $l_{1}$ minimization problem (1.7). Friedlander et al. [16] pointed out that once at least $50 \%$ of the support information is accurate, a less conservative sufficient condition for guaranteeing stably and robustly signal reconstruction as well as a tighter reconstruction error bound can be obtained. Furthermore, they also pointed out sufficient conditions are weaker than those of [24] when $\omega=0$.

In this paper, we consider the following weighted $l_{1}$ minimization method:

$$
\begin{aligned}
& \underset{x \in \mathbb{R}^{N}}{\operatorname{minimize}}\|x\|_{1, \mathrm{w}} \quad \text { subject to } y-A x \in \mathcal{B} \\
& \text { with } \mathrm{w}_{i}= \begin{cases}1, & i \in \widetilde{T}^{c} \\
\omega, & i \in \widetilde{T} .\end{cases}
\end{aligned}
$$

where $0 \leq \omega \leq 1$ and $\widetilde{T} \subset\{1,2, \ldots, N\}$ is a given support estimate of unknown signal $x$. $\mathcal{B}$ is also a bounded set determined by the noise settings (1.5) and (1.6). Our goal is to generalize the results of Cai and Zhang [7] via the weighted $l_{1}$ minimization method (1.8). We establish the sufficient condition on RIC and ROC for the stable and robust recovery of signals with partially known support information from (1.1). We also show that the recovery by weighted $l_{1}$ minimization method (1.8) is stable and robust under weaker sufficient conditions compared to the standard $l_{1}$ minimization method (1.4) when we have the partial support information with accuracy better than $50 \%$. Meanwhile, we obtain the smaller upper bounds on the reconstruction error under additional conditions. By means of weighted $l_{1}$ minimization method (1.8), that is to say, the requirement on the RIC and ROC of the sensing matrix for guaranteeing stable and robust signal recovery can be further relaxed if at least $50 \%$ of the support estimate is accurate; in addition, the reconstruction error upper bound is provably to be smaller under additional conditions. Our result implies that the achievable performance of signal recovery via weighted $l_{1}$ minimization method (1.8) is actually better than the works by Cai and Zhang [7] under some conditions.

The rest of the paper is organized as follows. In Section 2, we will introduce some notations and some basic lemmas that will be used. The main results are given in Section 3 , and the proofs 
of our main results are presented in Section 4 ,

\section{Preliminaries}

Let us begin with basic notations. For arbitrary $x \in \mathbb{R}^{N}, x_{\max (k)}$ is defined as $x$ with all but the largest $k$ entries in absolute value set to zero, i.e. $x_{\max (k)}$ is the best $k$-term approximation of $x$, and $x_{-\max (k)}=x-x_{\max (k)}$. Let $T_{0}$ be the support of $x_{\max (k)}$, with $T_{0} \subseteq\{1, \ldots, N\}$ and $\left|T_{0}\right| \leq k$. Let $\widetilde{T} \subseteq\{1, \ldots, N\}$ be the support estimate of $x$ with $|\widetilde{T}|=\rho k$, where $\rho \geq 0$ represents the ratio of the size of the estimated support to the size of the actual support of $x_{\max (k)}$ (or the support of $x$ if $x$ is $k$-sparse). Denote $\widetilde{T}_{\alpha}=T_{0} \cap \widetilde{T}$ and $\widetilde{T}_{\beta}=T_{0}^{c} \cap \widetilde{T}$ with $\left|\widetilde{T}_{\alpha}\right|=\alpha|\widetilde{T}|=\alpha \rho k$ and $\left|\widetilde{T}_{\beta}\right|=\beta|\widetilde{T}|=\beta \rho k$, where $\alpha$ denotes the ratio of the number of indices in $T_{0}$ that were accurately estimated in $\widetilde{T}$ to

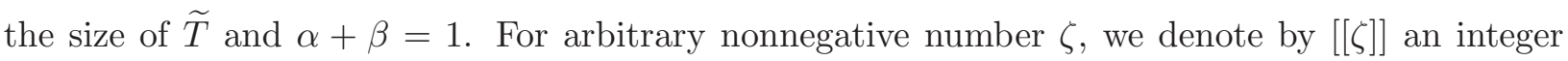
satisfying $\zeta \leq[[\zeta]]<\zeta+1$. Moreover, for given set $T \subseteq\{1, \ldots, N\}$, we denote by $x_{T}$ the vector which equals to $x$ on $T$ and 0 on the component $T^{c}$.

We first state three key technical tools used in the proof of the main result. Lemma 2.1 was introduced by Cai and Zhang ([7], Lemma 5.1) which provides a way to estimate the inner product by the ROC when only one component is sparse. Lemma 2.2 introduced by Cai and Zhang ([8], Lemma 5.3) provides an inequality between the sum of the $\alpha$ th power of two sequences of nonnegative numbers based on the inequality of their sums. Cai, Wang and Xu ([2], Lemma 1) supplied Lemma 2.3 that reveals the relationship between ROC's of different orders.

Lemma 2.1 ([7], Lemma 5.1). Let $k_{1}, k_{2} \leq N$ and $\lambda \geq 0$. Assume $u, v \in \mathbb{R}^{N}$ have disjoint supports and $u$ is $k_{1}$-sparse. If $\|v\|_{1} \leq \lambda k_{2}$ and $\|v\|_{\infty} \leq \lambda$, then

$$
|\langle A u, A v\rangle| \leq \theta_{k_{1}, k_{2}}\|u\|_{2} \cdot \lambda \sqrt{k_{2}} .
$$

Lemma 2.2 ([8], Lemma 5.3). Assume $m \geq k, a_{1} \geq a_{2} \geq \cdots \geq a_{m} \geq 0, \sum_{i=1}^{k} a_{i} \geq \sum_{i=k+1}^{m} a_{i}$, then for all $\alpha \geq 1$,

$$
\sum_{j=k+1}^{m} a_{j}^{\alpha} \leq \sum_{i=1}^{k} a_{i}^{\alpha}
$$

More generally, assume $a_{1} \geq a_{2} \geq \cdots \geq a_{m} \geq 0, \lambda \geq 0$ and $\sum_{i=1}^{k} a_{i}+\lambda \geq \sum_{i=k+1}^{m} a_{i}$, then for all $\alpha \geq 1$,

$$
\sum_{j=k+1}^{m} a_{j}^{\alpha} \leq k\left(\sqrt[\alpha]{\frac{\sum_{i=1}^{k} a_{i}^{\alpha}}{k}}+\frac{\lambda}{k}\right)^{\alpha}
$$


Lemma 2.3 ([2], Lemma 1). For any $\tau \geq 1$ and positive integers $k, k^{\prime}$ such that $\tau k^{\prime}$ is an integer, then

$$
\theta_{k, \tau k^{\prime}} \leq \sqrt{\tau} \theta_{k, k^{\prime}}
$$

As we mentioned in the introduction, Cai and Zhang [7] provided the sharp sufficient condition for ensuring exact and stable sparse signals reconstruction via $l_{1}$ minimization (1.4). Their main result can be stated as below.

Theorem 2.1 (7], Theorem 2.6). Let $y=A x+z$ with $\|z\|_{2} \leq \varepsilon$ and $\widehat{x}^{l_{2}}$ is the minimizer of (1.4) with $\mathcal{B}=\mathcal{B}^{l_{2}}(\eta)=\left\{z:\|z\|_{2} \leq \eta\right\}$ for some $\eta \geq \varepsilon$. If

$$
\delta_{a}+C_{a, b, k} \theta_{a, b}<1
$$

for some positive integers $a$ and $b$ with $1 \leq a \leq k$, where

$$
C_{a, b, k}=\max \left\{\frac{2 k-a}{\sqrt{a b}}, \sqrt{\frac{2 k-a}{a}}\right\}
$$

then

$$
\left\|\widehat{x}^{l_{2}}-x\right\|_{2} \leq C_{0}(\varepsilon+\eta)+C_{1} \cdot 2\left\|x_{-\max (k)}\right\|_{1},
$$

where

$$
C_{0}=\frac{\sqrt{2\left(1+\delta_{a}\right) k / a}}{1-\delta_{a}-C_{a, b, k} \theta_{a, b}}, \quad C_{1}=\frac{\sqrt{2 k} C_{a, b, k} \theta_{a, b}}{\left(1-\delta_{a}-C_{a, b, k} \theta_{a, b}\right)(2 k-a)}+\frac{1}{\sqrt{k}} .
$$

Theorem 2.2 (7], Theorem 2.7). Let $y=A x+z$ with $\left\|A^{T} z\right\|_{\infty} \leq \varepsilon$ and $\widehat{x}^{D S}$ is the minimizer of (1.4) with $\mathcal{B}=\mathcal{B}^{D S}(\eta)=\left\{z:\left\|A^{T} z\right\|_{\infty} \leq \eta\right\}$ for some $\eta \geq \varepsilon$. If $\delta_{a}+C_{a, b, k} \theta_{a, b}<1$ for some positive integers $a$ and $b$ with $1 \leq a \leq k$, where $C_{a, b, k}=\max \left\{\frac{2 k-a}{\sqrt{a b}}, \sqrt{\frac{2 k-a}{a}}\right\}$, then

$$
\left\|\widehat{x}^{D S}-x\right\|_{2} \leq C_{0}^{\prime}(\varepsilon+\eta)+C_{1}^{\prime} \cdot 2\left\|x_{-\max (k)}\right\|_{1},
$$

where

$$
C_{0}^{\prime}=\frac{\sqrt{2 k}}{1-\delta_{a}-C_{a, b, k} \theta_{a, b}}, \quad C_{1}^{\prime}=C_{1}
$$

Cai and Zhang pointed out that the sufficient condition (2.1) is sharp in Theorem 2.8 (see [7]). Namely, if $\delta_{a}+C_{a, b, k} \theta_{a, b}=1$, there does not exist any method that can exactly recover all $k$-sparse signals in noiseless case. Also, in noisy case, for any $\varepsilon>0, \delta_{a}+C_{a, b, k} \theta_{a, b}<1+\varepsilon$ can not guarantee the stable recovery of all $k$-sparse signals. 


\section{Main results}

Theorem 3.1. Let $x \in \mathbb{R}^{N}$ be an arbitrary signal and its best $k$-term approximation support on $T_{0} \subseteq\{1, \ldots, N\}$ with $\left|T_{0}\right| \leq k$. Let $\widetilde{T} \subseteq\{1, \ldots, N\}$ be an arbitrary set and denote $\rho \geq 0$ and $0 \leq \alpha \leq 1$ such that $|\widetilde{T}|=\rho k$ and $\left|\widetilde{T} \cap T_{0}\right|=\alpha \rho k$. Let $y=A x+z$ with $\|z\|_{2} \leq \varepsilon$ and $\widehat{x}^{l_{2}}$ is the minimizer of (1.8) with (1.5). If

$$
\delta_{a}+C_{a, b, k}^{\alpha, \omega} \theta_{a, b}<1
$$

for some positive integers $a$ and $b$ with $1 \leq a \leq k$, where

$$
C_{a, b, k}^{\alpha, \omega}=\max \left\{\frac{s}{\sqrt{a b}}, \sqrt{\frac{s}{a}}\right\}
$$

with

$$
s=[[k-a+\omega k+(1-\omega) \sqrt{(1+\rho-2 \alpha \rho) k} \cdot \max \{\sqrt{(1+\rho-2 \alpha \rho) k}, \sqrt{a}\}]] .
$$

Then

$$
\left\|\widehat{x}^{l_{2}}-x\right\|_{2} \leq D_{0}(2 \varepsilon)+D_{1} \cdot 2\left(\omega\left\|x_{T_{0}^{c}}\right\|_{1}+(1-\omega)\left\|x_{\widetilde{T}^{c} \cap T_{0}^{c}}\right\|_{1}\right)
$$

where

$$
\begin{aligned}
D_{0} & =\frac{\sqrt{2\left(1+\delta_{a}\right) d / a}}{1-\delta_{a}-C_{a, b, k}^{\alpha, \omega} \theta_{a, b}}, \\
D_{1} & =\frac{\sqrt{2 d} C_{a, b, s}^{\alpha, \omega} \theta_{a, b}}{\left(1-\delta_{a}-C_{a, b, k}^{\alpha, \omega} \theta_{a, b}\right) s}+\frac{1}{\sqrt{d}} .
\end{aligned}
$$

Let $y=A x+z$ with $\left\|A^{T} z\right\|_{\infty} \leq \varepsilon$. Assume that $\widehat{x}^{D S}$ is the minimizer of (1.8) with (1.6) and (3.1) holds. If

$$
\delta_{a}+C_{a, b, k}^{\alpha, \omega} \theta_{a, b}<1
$$

for some positive integers $a$ and $b$ with $1 \leq a \leq k$, where

$$
C_{a, b, k}^{\alpha, \omega}=\max \left\{\frac{s}{\sqrt{a b}}, \sqrt{\frac{s}{a}}\right\},
$$

where $s$ is given in (3.3). Then

$$
\left\|\widehat{x}^{D S}-x\right\|_{2} \leq D_{0}^{\prime}(2 \varepsilon)+D_{1}^{\prime} \cdot 2\left(\omega\left\|x_{T_{0}^{c}}\right\|_{1}+(1-\omega)\left\|x_{\widetilde{T}^{c} \cap T_{0}^{c}}\right\|_{1}\right),
$$

where

$$
D_{0}^{\prime}=\frac{\sqrt{2 d}}{1-\delta_{a}-C_{a, b, k}^{\alpha, \omega} \theta_{a, b}}, \quad D_{1}^{\prime}=D_{1} .
$$


Here

$$
d=\left\{\begin{array}{cc}
k, & \omega=1, \\
\max \{k,(1+\rho-2 \alpha \rho) k\}, & 0 \leq \omega<1 .
\end{array}\right.
$$

Remark 3.1. In Theorem 3.1, we observed that every signal $x \in \mathbb{R}^{N}$ can be stably and robustly recovered. And if $\mathcal{B}=\{0\}$ and $x$ is a $k$-sparse signal, then Theorem 3.1 ensures exact recovery of the signal $x$.

When the the measurement model (1.1) is with Gaussian noise, the above results on the bounded noise case can be directly applicable to the case where the noise is Gaussian by using the same argument as in 2, 5]. This is due to the fact Gaussian noise is essentially bounded. The concrete content is stated as follows.

Remark 3.2. Let $x \in \mathbb{R}^{N}$ be an arbitrary signal and its best $k$-term approximation support on $T_{0} \subseteq\{1, \ldots, N\}$ with $\left|T_{0}\right| \leq k$. Let $\widetilde{T} \subseteq\{1, \ldots, N\}$ be an arbitrary set and define $\rho \geq 0$ and $0 \leq \alpha \leq 1$ such that $|\widetilde{T}|=\rho k$ and $\left|\widetilde{T} \cap T_{0}\right|=\alpha \rho k$. Assume that $z \sim N_{n}\left(0, \sigma^{2} I\right)$ in (1.1) and $\delta_{a}+C_{a, b, k}^{\alpha, \omega} \theta_{a, b}<1$ for some positive integers a and $b$ with $1 \leq a \leq k$, where $C_{a, b, k}^{\alpha, \omega}=\max \left\{\frac{s}{\sqrt{a b}}, \sqrt{\frac{s}{a}}\right\}$ with $s=[[k-a+\omega k+(1-\omega) \sqrt{(1+\rho-2 \alpha \rho) k} \cdot \max \{\sqrt{(1+\rho-2 \alpha \rho) k}, \sqrt{a}\}]]$. Let $\mathcal{B}^{l_{2}}=\{z$ : $\left.\|z\|_{2} \leq \sigma \sqrt{n+2 \sqrt{n \log n}}\right\}$ and $\mathcal{B}^{D S}=\left\{z:\left\|A^{T} z\right\|_{\infty} \leq \sigma \sqrt{2 \log N}\right\} . \widehat{x}^{l_{2}}$ and $\widehat{x}^{D S}$ is the minimizer of (1.8) with $\mathcal{B}^{l_{2}}$ and $\mathcal{B}^{D S}$, respectively. Then, with probability at least $1-1 / n$,

$$
\left\|\widehat{x}^{l_{2}}-x\right\|_{2} \leq D_{0}(2 \sigma \sqrt{n+2 \sqrt{n \log n}})+D_{1} \cdot 2\left(\omega\left\|x_{T_{0}^{c}}\right\|_{1}+(1-\omega)\left\|x_{\widetilde{T}^{c} \cap T_{0}^{c}}\right\|_{1}\right),
$$

and

$$
\left\|\widehat{x}^{D S}-x\right\|_{2} \leq D_{0}^{\prime}(2 \sigma \sqrt{2 \log N})+D_{1}^{\prime} \cdot 2\left(\omega\left\|x_{T_{0}^{c}}\right\|_{1}+(1-\omega)\left\|x_{\widetilde{T}^{c} \cap T_{0}^{c}}\right\|_{1}\right),
$$

with probability at least $1-1 / \sqrt{\pi \log N}$.

Theorem 3.2. Let $1 \leq a \leq s \leq k, a+s \leq N$ and $b \geq 1$, where $s$ is defined as (3.3). Then there exists a sensing matrix $A \in \mathbb{R}^{n \times N}$ satisfying $\delta_{a}+C_{a, b, k}^{\alpha, \omega} \theta_{a, b}=1$ where $C_{a, b, k}^{\alpha, \omega}=\max \left\{\frac{s}{\sqrt{a b}}, \sqrt{\frac{s}{a}}\right\}$ and some $k$-sparse vector $\eta \in \mathbb{R}^{N}$ such that the weighted $l_{1}$ minimization method (1.8) fails to exactly recover the $k$-sparse vector $\eta$ in the noiseless case and stably recover the $k$-sparse vector $\eta$ in the noise case.

Remark 3.3. Theorem 3.2 implies that for arbitrarily $\varepsilon>0, \delta_{a}+C_{a, b, k}^{\alpha, \omega} \theta_{a, b}<1+\varepsilon$ is not sufficient to guarantee the exact recovery of all $k$-sparse vectors in noiseless case and the stable recovery of all $k$-sparse vectors in noise case.

Proposition 3.1. Let $s$ be defined as (3.3) and $d$ be defined as (3.8). 
(1) If $\omega=1$, then $s=2 k-a, d=k$. The sufficient condition (3.1) of Theorem 3.1 is identical to that of Theorem 2.1 and Theorem 2.2 with (2.1), and $D_{0}=C_{0}, D_{1}=C_{1}, D_{0}^{\prime}=C_{0}^{\prime}, D_{1}^{\prime}=C_{1}^{\prime}$. Moreover, the condition is sharp.

(2) If $\alpha=\frac{1}{2}$, then $s=2 k-a$ and $d=k$. The sufficient condition (3.1) of Theorem 3.1 is identical to that of Theorem 2.1 and Theorem 2.2 with (2.1), and $D_{0}=C_{0}, D_{1}=C_{1}, D_{0}^{\prime}=C_{0}^{\prime}, D_{1}^{\prime}=C_{1}^{\prime}$. Moreover, the condition is sharp.

(3) Assume $0 \leq \omega<1$. If $\alpha>\frac{1}{2}$, then $s<2 k-a$ and $d=k$. The sufficient condition (3.1) in Theorem 3.1 is weaker than that of Theorem 2.1 and Theorem 2.2 with (2.1), and $D_{0}<$ $C_{0}, D_{0}^{\prime}<C_{0}^{\prime}$.

(4) Suppose $0 \leq \omega<1$. If $\alpha>\frac{1}{2}$ and $b \leq s$, then $D_{1}<C_{1}$.

(5) Suppose $0 \leq \omega<1$. If $\alpha>\frac{1}{2}$ and $s<b \leq 2 k-a$, then $D_{1}<C_{1}$ if and only if $1-\delta_{a}-C_{a, b, k}^{\alpha, \omega} \theta_{a, b}<$ $\frac{2 k-a-\sqrt{b s}}{\sqrt{a}(\sqrt{b}-\sqrt{s})} \theta_{a, b}$.

(6) Suppose $0 \leq \omega<1$. If $\alpha>\frac{1}{2}$ and $b>2 k-a$, then $D_{1}<C_{1}$ if and only if $1-\delta_{a}-C_{a, b, k}^{\alpha, \omega} \theta_{a, b}<$ $\sqrt{\frac{2 k-a}{a}} \theta_{a, b}$

\section{Proofs}

Proof of Theorem 3.1. Firstly, we show the estimate (3.4). Let $h=\widehat{x}^{l_{2}}-x$, where $x$ is the original signal and $\widehat{x}^{l_{2}}$ is the minimizer of (1.8) with (1.5). We can express $h$ as $h=\sum_{i=1}^{N} c_{i} u_{i}$, where $\left\{c_{i}\right\}_{i=1}^{N}$ are nonnegative and decreasing, i.e. $c_{1} \geq c_{2} \geq \cdots \geq c_{N} \geq 0,\left\{u_{i}\right\}_{i=1}^{N}$ are different unit vectors with one entry of \pm 1 and other entries of zeros. From the following inequality proved by Friedlander et al. (see (21) in [16])

$$
\left\|h_{T_{0}^{c}}\right\|_{1} \leq \omega\left\|h_{T_{0}}\right\|_{1}+(1-\omega)\left\|h_{T_{0} \cup \widetilde{T} \backslash \widetilde{T}_{\alpha}}\right\|_{1}+2\left(\omega\left\|x_{T_{0}^{c}}\right\|_{1}+(1-\omega)\left\|x_{\widetilde{T}^{c} \cap T_{0}^{c}}\right\|_{1}\right),
$$

we have

$$
\sum_{i=k+1}^{N} c_{i}=\left\|h_{-\max (k)}\right\|_{1} \leq \omega\left\|h_{T_{0}}\right\|_{1}+(1-\omega)\left\|h_{T_{0} \cup \widetilde{T} \backslash \widetilde{T}_{\alpha}}\right\|_{1}+2\left(\omega\left\|x_{T_{0}^{c}}\right\|_{1}+(1-\omega)\left\|x_{\widetilde{T}^{c} \cap T_{0}^{c}}\right\|_{1}\right) .
$$

Noting that $\left|T_{0} \cup \widetilde{T} \backslash \widetilde{T}_{\alpha}\right|=(1+\rho-2 \alpha \rho) k$, thus

$$
\left\|h_{-\max (a)}\right\|_{\infty}=c_{a+1} \leq \frac{\sum_{i=1}^{a} c_{i}}{a}=\frac{\left\|h_{\max (a)}\right\|_{1}}{a} \leq \frac{\left\|h_{\max (a)}\right\|_{2}}{\sqrt{a}}
$$




$$
\begin{aligned}
\left\|h_{-\max (a)}\right\|_{1}= & \sum_{i=a+1}^{k} c_{i}+\sum_{i=k+1}^{N} c_{i} \\
\leq & \frac{k-a}{k} \sum_{i=1}^{k} c_{i}+\omega\left\|h_{T_{0}}\right\|_{1}+(1-\omega)\left\|h_{T_{0} \cup \widetilde{T} \backslash \widetilde{T}_{\alpha}}\right\|_{1}+2\left(\omega\left\|x_{T_{0}^{c}}\right\|_{1}+(1-\omega)\left\|x_{\widetilde{T}^{c} \cap T_{0}^{c}}\right\|_{1}\right) \\
\leq & \frac{k-a}{a}\left\|h_{\max (a)}\right\|_{1}+\omega \sqrt{k}\left\|h_{T_{0}}\right\|_{2}+(1-\omega) \sqrt{(1+\rho-2 \alpha \rho) k}\left\|h_{T_{0} \cup \widetilde{T} \backslash \widetilde{T}_{\alpha}}\right\|_{2} \\
& +2\left(\omega\left\|x_{T_{0}^{c}}\right\|_{1}+(1-\omega)\left\|x_{\widetilde{T}^{c} \cap T_{0}^{c}}\right\|_{1}\right) \\
\leq & \frac{k-a}{\sqrt{a}}\left\|h_{\max (a)}\right\|_{2}+\omega \sqrt{k} \sqrt{\frac{k}{a}}\left\|h_{\max (a)}\right\|_{2} \\
& +(1-\omega) \sqrt{(1+\rho-2 \alpha \rho) k} \cdot \max \left\{\sqrt{\frac{(1+\rho-2 \alpha \rho) k}{a}}, 1\right\}\left\|h_{\max (a)}\right\|_{2} \\
& +2\left(\omega\left\|x_{T_{0}^{c}}\right\|_{1}+(1-\omega)\left\|x_{\widetilde{T}^{c} \cap T_{0}^{c}}\right\|_{1}\right) \\
= & (k-a+\omega k+(1-\omega) \sqrt{(1+\rho-2 \alpha \rho) k} \cdot \max \{\sqrt{(1+\rho-2 \alpha \rho) k}, \sqrt{a}\}) \frac{\left\|h_{\max (a)}\right\|_{2}}{\sqrt{a}} \\
& +2\left(\omega\left\|x_{T_{0}^{c}}\right\|_{1}+(1-\omega)\left\|x_{\widetilde{T}^{c} \cap T_{0}^{c}}\right\|_{1}\right) \\
\leq & \frac{\left\|h_{\max (a)}\right\|_{2}}{\sqrt{a}}+2\left(\omega\left\|x_{T_{0}^{c}}\right\|_{1}+(1-\omega)\left\|x_{\widetilde{T}^{c} \cap T_{0}^{c}}\right\|_{1}\right),
\end{aligned}
$$

where $s=[[k-a+\omega k+(1-\omega) \sqrt{(1+\rho-2 \alpha \rho) k} \cdot \max \{\sqrt{(1+\rho-2 \alpha \rho) k}, \sqrt{a}\}]]$. Taking $k_{1}=$ $a, k_{2}=s, \lambda=\frac{\left\|h_{\max (a)}\right\|_{2}}{\sqrt{a}}+\frac{2\left(\omega\left\|x_{T_{0}^{c}}\right\|_{1}+(1-\omega)\left\|x_{\widetilde{T}^{c} \cap T_{0}^{c}}\right\|_{1}\right)}{s}$, from above inequalities and Lemma 2.1], we obtain

$$
\left|\left\langle A h_{\max (a)}, A h_{-\max (a)}\right\rangle\right| \leq \theta_{a, s}\left\|h_{\max (a)}\right\|_{2} \sqrt{s} \cdot\left(\frac{\left\|h_{\max (a)}\right\|_{2}}{\sqrt{a}}+\frac{2\left(\omega\left\|x_{T_{0}^{c}}\right\|_{1}+(1-\omega)\left\|x_{\widetilde{T}^{c} \cap T_{0}^{c}}\right\|_{1}\right)}{s}\right) .
$$

Combining the definition of $\delta_{k}$ and the fact that

$$
\|A h\|_{2}=\left\|A \widehat{x}^{l_{2}}-A x\right\|_{2} \leq\left\|y-A \widehat{x}^{l_{2}}\right\|_{2}+\|A x-y\|_{2} \leq 2 \varepsilon
$$

we have

$$
\begin{aligned}
\left|\left\langle A h_{\max (a)}, A h\right\rangle\right| & \leq\left\|A h_{\max (a)}\right\|_{2}\|A h\|_{2} \\
& \leq \sqrt{1+\delta_{a}}\left\|h_{\max (a)}\right\|_{2} \cdot(2 \varepsilon) .
\end{aligned}
$$


Hence,

$$
\begin{aligned}
(2 \varepsilon) & \sqrt{1+\delta_{a}}\left\|h_{\max (a)}\right\|_{2} \geq\left|\left\langle A h_{\max (a)}, A h\right\rangle\right| \\
& \geq\left\|A h_{\max (a)}\right\|_{2}^{2}-\left|\left\langle A h_{\max (a)}, A h_{-\max (a)}\right\rangle\right| \\
& \geq\left(1-\delta_{a}\right)\left\|h_{\max (a)}\right\|_{2}^{2}-\theta_{a, s}\left\|h_{\max (a)}\right\|_{2} \sqrt{s} \cdot\left(\frac{\left\|h_{\max (a)}\right\|_{2}}{\sqrt{a}}+\frac{2\left(\omega\left\|x_{T_{0}^{c}}\right\|_{1}+(1-\omega)\left\|x_{\widetilde{T}^{c} \cap T_{0}^{c}}\right\|_{1}\right)}{s}\right) \\
& =\left(1-\delta_{a}-\sqrt{\frac{s}{a}} \theta_{a, s}\right)\left\|h_{\max (a)}\right\|_{2}^{2}-\theta_{a, s}\left\|h_{\max (a)}\right\|_{2} \frac{2\left(\omega\left\|x_{T_{0}^{c}}\right\|_{1}+(1-\omega)\left\|x_{\widetilde{T}^{c} \cap T_{0}^{c}}\right\|_{1}\right)}{\sqrt{s}} .
\end{aligned}
$$

It follows from the above inequality that

$$
\left\|h_{\max (a)}\right\|_{2} \leq \frac{\sqrt{1+\delta_{a}}(2 \varepsilon)}{1-\delta_{a}-\sqrt{\frac{s}{a}} \theta_{a, s}}+\frac{\theta_{a, s}}{1-\delta_{a}-\sqrt{\frac{s}{a}} \theta_{a, s}} \frac{2\left(\omega\left\|x_{T_{0}^{c}}\right\|_{1}+(1-\omega)\left\|x_{\widetilde{T}^{c} \cap T_{0}^{c}}\right\|_{1}\right)}{\sqrt{s}} .
$$

Define

$$
d=\left\{\begin{array}{cc}
k, & \omega=1 \\
\max \{k,(1+\rho-2 \alpha \rho) k\}, & 0 \leq \omega<1 .
\end{array}\right.
$$

With (4.1), it is clear that

$$
\left\|h_{-\max (d)}\right\|_{1} \leq\left\|h_{\max (d)}\right\|_{1}+2\left(\omega\left\|x_{T_{0}^{c}}\right\|_{1}+(1-\omega)\left\|x_{\widetilde{T}^{c} \cap T_{0}^{c}}\right\|_{1}\right) .
$$

From Lemma 2.2, we have

$$
\left\|h_{-\max (d)}\right\|_{2} \leq\left\|h_{\max (d)}\right\|_{2}+\frac{2\left(\omega\left\|x_{T_{0}^{c}}\right\|_{1}+(1-\omega)\left\|x_{\widetilde{T}^{c} \cap T_{0}^{c}}\right\|_{1}\right)}{\sqrt{d}} .
$$


Therefore,

$$
\begin{aligned}
\|h\|_{2} & =\sqrt{\left\|h_{\max (d)}\right\|_{2}^{2}+\left\|h_{-\max (d)}\right\|_{2}^{2}} \\
& \leq \sqrt{\left\|h_{\max (d)}\right\|_{2}^{2}+\left(\left\|h_{\max (d)}\right\|_{2}+\frac{2\left(\omega\left\|x_{T_{0}^{c}}\right\|_{1}+(1-\omega)\left\|x_{\widetilde{T}^{c} \cap T_{0}^{c}}\right\|_{1}\right)}{\sqrt{d}}\right)^{2}} \\
& \leq \sqrt{2\left\|h_{\max (d)}\right\|_{2}^{2}}+\frac{2\left(\omega\left\|x_{T_{0}^{c}}\right\|_{1}+(1-\omega)\left\|x_{\widetilde{T}^{c} \cap T_{0}^{c}}\right\|_{1}\right)}{\sqrt{d}} \\
& =\sqrt{2 \sum_{i=1}^{d} c_{i}^{2}+\frac{2\left(\omega\left\|x_{T_{0}^{c}}\right\|_{1}+(1-\omega)\left\|x_{\widetilde{T}^{c} \cap T_{0}^{c}}\right\|_{1}\right)}{\sqrt{d}}} \\
& \leq \sqrt{\frac{2 d}{a} \sum_{i=1}^{a} c_{i}^{2}+\frac{2\left(\omega\left\|x_{T_{0}^{c}}\right\|_{1}+(1-\omega)\left\|x_{\widetilde{T}^{c} \cap T_{0}^{c}}\right\|_{1}\right)}{\sqrt{d}}} \\
& =\sqrt{\frac{2 d}{a}\left\|h_{\max (a)}\right\|_{2}+\frac{2\left(\omega\left\|x_{T_{0}^{c}}\right\|_{1}+(1-\omega)\left\|x_{\widetilde{T}^{c} \cap T_{0}^{c}}\right\|_{1}\right)}{\sqrt{d}}} \\
& \leq \frac{\sqrt{2\left(1+\delta_{a}\right) d / a}}{1-\delta_{a}-\sqrt{\frac{s}{a}} \theta_{a, s}}(2 \varepsilon)+\left(\frac{\sqrt{2 d / a} \theta_{a, s}}{\left(1-\delta_{a}-\sqrt{\frac{s}{a}} \theta_{a, s}\right) \sqrt{s}}+\frac{1}{\sqrt{d}}\right) \cdot 2\left(\omega\left\|x_{T_{0}^{c}}\right\|_{1}+(1-\omega)\left\|x_{\widetilde{T}^{c} \cap T_{0}^{c}}\right\|_{1}\right) .
\end{aligned}
$$

Since

$$
\theta_{a, s}=\theta_{a, \frac{s}{\min \{b, s\}}} \min \{b, s\} \leq \sqrt{\frac{s}{\min \{b, s\}}} \theta_{a, \min \{b, s\}} \leq \max \left\{\sqrt{\frac{s}{b}}, 1\right\} \theta_{a, b}=\sqrt{\frac{a}{s}} C_{a, b, k}^{\alpha, \omega} \theta_{a, b},
$$

where $C_{a, b, k}^{\alpha, \omega}=\max \left\{\frac{s}{\sqrt{a b}}, \sqrt{\frac{s}{a}}\right\}$, and the first inequality follows from Lemma 2.3. Consequently,

$$
\|h\|_{2} \leq \frac{\sqrt{2\left(1+\delta_{a}\right) d / a}}{1-\delta_{a}-C_{a, b, k}^{\alpha, \omega} \theta_{a, b}}(2 \varepsilon)+\left(\frac{\sqrt{2 d} C_{a, b, k}^{\alpha, \omega} \theta_{a, b}}{\left(1-\delta_{a}-C_{a, b, k}^{\alpha, \omega} \theta_{a, b}\right) s}+\frac{1}{\sqrt{d}}\right) \cdot 2\left(\omega\left\|x_{T_{0}^{c}}\right\|_{1}+(1-\omega)\left\|x_{\widetilde{T}^{c} \cap T_{0}^{c}}\right\|_{1}\right)
$$

So, (3.4) is obtained.

Next, we can prove (3.6) going along similar lines to that of (3.4). To prove( (3.6), we only need to use the following (4.4) and (4.5) instead of (4.2) and (4.3), respectively.

$$
\begin{aligned}
\left\|A^{T} A h\right\|_{\infty} & =\left\|A^{T} A\left(\widehat{x}^{D S}-x\right)\right\|_{\infty} \\
& \leq\left\|A^{T}\left(A \widehat{x}^{D S}-y\right)\right\|_{\infty}+\left\|A^{T}(y-A x)\right\|_{\infty} \\
& \leq 2 \varepsilon, \\
\left|\left\langle A h_{\max (a)}, A h\right\rangle\right| & =\left|\left\langle h_{\max (a)}, A^{T} A h\right\rangle\right| \\
& \leq\left\|h_{\max (a)}\right\|_{1}\left\|A^{T} A h\right\|_{\infty} \\
& \leq \sqrt{a}\left\|h_{\max (a)}\right\|_{2} \cdot(2 \varepsilon) .
\end{aligned}
$$


This completes the proof of Theorem 3.1 .

Proof of Theorem 3.2. Firstly, let $L=a+s$, and

$$
\begin{aligned}
\xi_{1} & =\frac{1}{\sqrt{L}}(\overbrace{1, \ldots, 1}^{L}, 0, \ldots, 0) \in \mathbb{R}^{N}, \quad \text { if } L-k>\rho k, \\
\text { or } \quad \xi_{1} & =\frac{1}{\sqrt{L}}(\underbrace{1, \ldots, 1}_{k-\alpha \rho k}, \underbrace{\overbrace{1, \ldots, 1}^{L-k}, 0, \ldots, 0}_{\rho k}, \underbrace{1, \ldots, 1}_{\alpha \rho k}, 0, \ldots, 0) \in \mathbb{R}^{N}, \quad \text { if } L-k \leq \rho k,
\end{aligned}
$$

Due to $\left\|\xi_{1}\right\|_{2}=1$, we extend $\xi_{1}$ into an orthonormal basis $\left\{\xi_{1}, \ldots, \xi_{N}\right\}$ of $\mathbb{R}^{N}$. Next, we define the linear map $A: \mathbb{R}^{N} \rightarrow \mathbb{R}^{N}$ such that for all $x=\sum_{i=1}^{N} c_{i} \xi_{i} \in \mathbb{R}^{N}$,

$$
A x=\sqrt{1+\frac{L-s}{L+s}}\left(x-\left\langle\xi_{1}, x\right\rangle \xi_{1}\right)=\sqrt{1+\frac{L-s}{L+s}} \sum_{i=2}^{N} c_{i} \xi_{i} .
$$

Then for any $a$-sparse signal $x$, we can easily gain

$$
\|A x\|_{2}^{2}=\left(1+\frac{L-s}{L+s}\right)\left(\|x\|_{2}^{2}-\left|\left\langle\xi_{1}, x\right\rangle\right|^{2}\right)
$$

and

$$
\left|\left\langle\xi_{1}, x\right\rangle\right|^{2} \leq\|x\|_{2}^{2} \cdot \sum_{i \in \operatorname{supp}(x)}\left|\xi_{1}(i)\right|^{2} \leq\|x\|_{2}^{2} \cdot\left\|\xi_{1, \max (a)}\right\|_{2}^{2} \leq \frac{a}{L}\|x\|_{2}^{2}=\frac{L-s}{L}\|x\|_{2}^{2} .
$$

Hence,

$$
\left(1+\frac{L-s}{L+s}\right)\|x\|_{2}^{2} \geq\|A x\|_{2}^{2} \geq\left(1+\frac{L-s}{L+s}\right)\left(1-\frac{L-s}{L}\right)\|x\|_{2}^{2}=\left(1-\frac{L-s}{L+s}\right)\|x\|_{2}^{2},
$$

which deduces

$$
\delta_{a} \leq \frac{L-s}{L+s} .
$$

Finally, we estimate $\theta_{a, b}$. For arbitrary $a$-sparse vector $u \in \mathbb{R}^{N}$ and $b$-sparse vector $v \in \mathbb{R}^{N}$ with disjoint supports, we define $u=\sum_{i=1}^{N} l_{i} \xi_{i}$ and $v=\sum_{i=1}^{N} d_{i} \xi_{i}$. It follows immediately that $0=\langle u, v\rangle=\sum_{i=1}^{N} l_{i} d_{i}$.

(i) When $b \leq s$, through a simple calculation, it can be concluded that

$$
\left|l_{1}\right|=\left|\left\langle\xi_{1}, u\right\rangle\right| \leq\|u\|_{2} \cdot\left(\sum_{i \in \operatorname{supp}(u)}\left|\xi_{1}(i)\right|^{2}\right)^{1 / 2} \leq\|u\|_{2} \cdot\left\|\xi_{1, \max (a)}\right\|_{2} \leq \sqrt{\frac{a}{L}}\|u\|_{2},
$$

and

$$
\left|d_{1}\right|=\left|\left\langle\xi_{1}, v\right\rangle\right| \leq\|v\|_{2} \cdot\left(\sum_{i \in \operatorname{supp}(v)}\left|\xi_{1}(i)\right|^{2}\right)^{1 / 2} \leq\|v\|_{2} \cdot\left\|\xi_{1, \max (b)}\right\|_{2} \leq \sqrt{\frac{b}{L}}\|v\|_{2} .
$$


It then follows that

$$
\frac{1}{1+\frac{L-s}{L+s}}|\langle A u, A v\rangle|=\left|\sum_{i=2}^{N} l_{i} d_{i}\right|=\left|-l_{1} d_{1}\right| \leq \frac{\sqrt{a b}}{L}\|u\|_{2}\|v\|_{2} .
$$

Accordingly,

$$
\theta_{a, b} \leq\left(1+\frac{L-s}{L+s}\right) \frac{\sqrt{a b}}{L}
$$

Therefore,

$$
\begin{aligned}
\delta_{a}+C_{a, b, k}^{\alpha, \omega} \theta_{a, b} & \leq \frac{L-s}{L+s}+\max \left\{\frac{s}{\sqrt{a b}}, \sqrt{\frac{s}{a}}\right\} \cdot\left(1+\frac{L-s}{L+s}\right) \frac{\sqrt{a b}}{L} \\
& =\frac{L-s}{L+s}+\frac{s}{\sqrt{a b}}\left(1+\frac{L-s}{L+s}\right) \frac{\sqrt{a b}}{L} \\
& =1 .
\end{aligned}
$$

(ii) When $b>s$, without loss of generality, we can suppose that $u$ and $v$ are nonzero. If $u=0$ or $v=0$, clearly $\langle A u, A v\rangle=0 \leq C\|u\|_{2}\|v\|_{2}$ holds for all $C>0$. We normalize $u$ and $v$ such that $\|u\|_{2}=\|v\|_{2}=1$. Because $u$ is $a$-sparse and $v$ is $b$-sparse, and $u, v$ have disjoint supports, we conclude

$$
\left|l_{1}\right|=\left|\left\langle\xi_{1}, u\right\rangle\right| \leq \sqrt{\frac{a}{L}}\|u\|_{2}=\sqrt{\frac{a}{L}}=\sqrt{\frac{a}{s+a}}
$$

and

$$
\begin{aligned}
\left|d_{1} \pm \sqrt{\frac{a}{s}} l_{1}\right| & =\left|\left\langle\xi_{1}, v \pm \sqrt{\frac{a}{s}} u\right\rangle\right| \leq\left\|v \pm \sqrt{\frac{a}{s}} u\right\|_{2} \\
& =\sqrt{\|v\|_{2}^{2}+\frac{a}{s}\|u\|_{2}^{2}}=\sqrt{\frac{s+a}{s}} .
\end{aligned}
$$


In view of $\left|l_{1}\right| \leq \sqrt{\frac{a}{a+s}}$ and $1 \leq a \leq s$,

$$
\begin{aligned}
\frac{1}{1+\frac{L-s}{L+s}}|\langle A u, A v\rangle| & =\left|\sum_{i=2}^{N} l_{i} d_{i}\right|=\left|-l_{1} d_{1}\right| \\
& =\left(\max \left\{\left|d_{1}+\sqrt{\frac{a}{s}} l_{1}\right|,\left|d_{1}-\sqrt{\frac{a}{s}} l_{1}\right|\right\}-\left|\sqrt{\frac{a}{s}} l_{1}\right|\right) \cdot\left|l_{1}\right| \\
& \leq\left|l_{1}\right|\left(\sqrt{\frac{s+a}{s}}-\sqrt{\frac{a}{s}}\left|l_{1}\right|\right) \\
& =-\sqrt{\frac{a}{s}}\left(\left|l_{1}\right|^{2}-\sqrt{\frac{s+a}{a}}\left|l_{1}\right|\right) \\
& =-\sqrt{\frac{a}{s}}\left(\left|l_{1}\right|-\frac{1}{2} \sqrt{\frac{s+a}{a}}\right)^{2}+\frac{s+a}{4 \sqrt{a s}} \\
& \leq-\sqrt{\frac{a}{s}}\left(\sqrt{\frac{a}{s+a}}-\frac{1}{2} \sqrt{\frac{s+a}{a}}\right)^{2}+\frac{s+a}{4 \sqrt{a s}} \\
& =\frac{\sqrt{a s}}{s+a}=\frac{\sqrt{a s}}{L}
\end{aligned}
$$

which implies

$$
\theta_{a, b} \leq\left(1+\frac{L-s}{L+s}\right) \frac{\sqrt{a s}}{L}
$$

Hence,

$$
\begin{aligned}
\delta_{a}+C_{a, b, k}^{\alpha, \omega} \theta_{a, b} & \leq \frac{L-s}{L+s}+\max \left\{\frac{s}{\sqrt{a b}}, \sqrt{\frac{s}{a}}\right\} \cdot\left(1+\frac{L-s}{L+s}\right) \frac{\sqrt{a s}}{L} \\
& =\frac{L-s}{L+s}+\sqrt{\frac{s}{a}}\left(1+\frac{L-s}{L+s}\right) \frac{\sqrt{a s}}{L} \\
& =1 .
\end{aligned}
$$

In a word, $\delta_{a}+C_{a, b, k}^{\alpha, \omega} \theta_{a, b} \leq 1$ has been proved.

Next, we define

$$
\begin{aligned}
\eta & =(\overbrace{1, \ldots, 1}^{k-\alpha \rho k}, \overbrace{0, \ldots, 0}^{\overbrace{0, \ldots}^{\rho k}}, \overbrace{1, \ldots, 1}^{\alpha \rho k}, 0, \ldots, 0) \in \mathbb{R}^{N}, \\
\gamma & =(\underbrace{0, \ldots, 0}_{k-\alpha \rho k}, \underbrace{-1, \ldots,-1}_{\rho k} \underbrace{0, \ldots, 0}_{\alpha \rho k}, \underbrace{-1, \ldots,-1}_{L-k-\rho k}, 0, \ldots, 0) \in \mathbb{R}^{N}, \quad \text { if } L-k>\rho k, \\
\text { or } \quad \gamma & =(\underbrace{0, \ldots, 0}_{k-\alpha \rho k}, \underbrace{\overbrace{-1, \ldots,-1}^{L-k}, 0, \ldots, 0}_{\rho k}, \underbrace{0, \ldots, 0}_{\alpha \rho k}, 0, \ldots, 0) \in \mathbb{R}^{N}, \quad \text { if } L-k \leq \rho k .
\end{aligned}
$$


From $1 \leq a \leq s \leq k$ and $L=a+s$, we have $L-k \leq k$. Hence $\eta$ and $\gamma$ are $k$-sparse. Moreover, $\|\eta\|_{1, \mathrm{w}}=k,\|\gamma\|_{1, \mathrm{w}} \leq L-k \leq k$. Note that $\|\gamma\|_{1, \mathrm{w}} \leq\|\eta\|_{1, \mathrm{w}}$ and $\xi_{1}=\frac{1}{\sqrt{L}}(\eta-\gamma)$. Since $A \xi_{1}=0$, we obtain $A \eta=A \gamma$.

(i) $\|\gamma\|_{1, \mathrm{w}}<\|\eta\|_{1, \mathrm{w}}$.

In the noiseless case $y=A \eta$, if weighted $l_{1}$ minimization method (1.8) can exactly recover $\eta$, namely, $\widehat{\eta}=\eta$. Clearly, $\|\widehat{\eta}\|_{1, \mathrm{w}}=\|\eta\|_{1, \mathrm{w}}$. It contradicts that $\|\gamma\|_{1, \mathrm{w}}<\|\eta\|_{1, \mathrm{w}}$.

In the noise case $y=A \eta+z$, suppose weighted $l_{1}$ minimization method (1.8) can stable recover $\eta$ with constraint $\mathcal{B}$, i.e., $\lim _{z \rightarrow 0} \widehat{\eta}=\eta$. Due to $y-A(\widehat{\eta}-\eta+\gamma)=y-A \widehat{\eta} \in \mathcal{B}$ and the definition of $\widehat{\eta}$, it follows immediately that $\|\widehat{\eta}\|_{1, \mathrm{w}} \leq\|\widehat{\eta}-\eta+\gamma\|_{1, \mathrm{w}}$. Thus, we have $\|\eta\|_{1, \mathrm{w}} \leq\|\gamma\|_{1, \mathrm{w}}$ as $z \rightarrow 0$. It contradicts that $\|\gamma\|_{1, \mathrm{w}}<\|\eta\|_{1, \mathrm{w}}$.

(ii) $\|\gamma\|_{1, \mathrm{w}}=\|\eta\|_{1, \mathrm{w}}$. The weighted $l_{1}$ method (1.8) does not distinguish $k$-sparse signals $\eta$ and $\gamma$ based $y$ and $A$.

Hence the weighted $l_{1}$ method (1.8) does not exactly and stably recover the $k$-sparse signal $\eta$ based on $A$ and $y$. Combining Theorem 3.1, we have $\delta_{a}+C_{a, b, k}^{\alpha, \omega} \theta_{a, b}=1$. This completes the proof of the theorem.

Proof of Proposition 3.1. For (1) and (2), when $\omega=1$ or $\alpha=\frac{1}{2}$, by simple calculation, we have $s=2 k-a, d=k$. Then, it is easy to imply (1) and (2) by comparing Theorem 3.1 with Theorem 2.1 and Theorem 2.2 ,

(3) Let $0 \leq \omega<1$. If $\alpha>\frac{1}{2}$, by means of the definition of $s$ in (3.3) and $d$ in (3.8), it follows immediately that $s<2 k-a, d=k$.

When $b \leq s, C_{a, b, k}^{\alpha, \omega}=\frac{s}{\sqrt{a b}}<\frac{2 k-a}{\sqrt{a b}}=C_{a, b, k}$.

When $s<b \leq 2 k-a, C_{a, b, k}^{\alpha, \omega}=\sqrt{\frac{s}{a}}<\frac{2 k-a}{\sqrt{a b}}=C_{a, b, k}$.

When $b \geq 2 k-a, C_{a, b, k}^{\alpha, \omega}=\sqrt{\frac{s}{a}}<\sqrt{\frac{2 k-a}{a}}=C_{a, b, k}$.

For any positive integers $a$ and $b$ with $1 \leq a \leq k$, in short, we obtain $C_{a, b, k}^{\alpha, \omega}<C_{a, b, k}$, which implies $\delta_{a}+C_{a, b, k}^{\alpha, \omega} \theta_{a, b}<\delta_{a}+C_{a, b, k} \theta_{a, b}$. Thus the condition $\delta_{a}+C_{a, b, k}^{\alpha, \omega} \theta_{a, b}<1$ in (3.1) is weaker than $\delta_{a}+C_{a, b, k} \theta_{a, b}<1$ in (2.1) and $D_{0}=\frac{\sqrt{2\left(1+\delta_{a}\right) k / a}}{1-\delta_{a}-C_{a, b, k}^{\alpha, \omega} \theta_{a, b}}<\frac{\sqrt{2\left(1+\delta_{a}\right) k / a}}{1-\delta_{a}-C_{a, b, k} \theta_{a, b}}=C_{0}, D_{0}^{\prime}=\frac{\sqrt{2 k}}{1-\delta_{a}-C_{a, b, k}^{\alpha, \omega} \theta_{a, b}}<$ $\frac{\sqrt{2 k}}{1-\delta_{a}-C_{a, b, k} \theta_{a, b}}=C_{0}^{\prime}$, which implies (3).

(4) Assume $0 \leq \omega<1$. If $\alpha>\frac{1}{2}$ and $b \leq s$, we have $d=k$ and $\delta_{a}+C_{a, b, k}^{\alpha, \omega} \theta_{a, b}<\delta_{a}+C_{a, b, k} \theta_{a, b}$. Combining the definition of $C_{1}$ and $D_{1}$, obviously, $D_{1}=\frac{\sqrt{2 k} \frac{1}{\sqrt{a b}} \theta_{a, b}}{1-\delta_{a}-C_{a, b, k}^{\alpha, \omega} \theta_{a, b}}+\frac{1}{\sqrt{k}}<\frac{\sqrt{2 k} \frac{1}{\sqrt{a b}} \theta_{a, b}}{1-\delta_{a}-C_{a, b, k} \theta_{a, b}}+\frac{1}{\sqrt{k}}=$ $C_{1}$.

(5) Since $\alpha>\frac{1}{2}$ and $s<b \leq 2 k-a, C_{a, b, k}^{\alpha, \omega}=\sqrt{\frac{s}{a}}, C_{a, b, k}=\frac{2 k-a}{\sqrt{a b}}$. Thus, to prove $D_{1}<C_{1}$, we just need to prove $\frac{\sqrt{2 k} \sqrt{\frac{1}{a}} \theta_{a, b}}{\left(1-\delta_{a}-\sqrt{\frac{s}{a}} \theta_{a, b}\right) \sqrt{s}}+\frac{1}{\sqrt{k}}<\frac{\sqrt{2 k} \sqrt{\frac{1}{a b}} \theta_{a, b}}{1-\delta_{a}-\frac{2 k-a}{\sqrt{a b}} \theta_{a, b}}+\frac{1}{\sqrt{k}}$. It is equal to prove that $1-\delta_{a}<\frac{2 k-a-s}{\sqrt{a}(\sqrt{b}-\sqrt{s})} \theta_{a, b}$, namely, $1-\delta_{a}-C_{a, b, k}^{\alpha, \omega} \theta_{a, b}<\frac{2 k-a-\sqrt{b s}}{\sqrt{a}(\sqrt{b}-\sqrt{s})} \theta_{a, b}$. 
(6) Due to $\alpha>\frac{1}{2}$ and $b>2 k-a$, we have $C_{a, b, k}^{\alpha, \omega}=\sqrt{\frac{s}{a}}$ and $C_{a, b, k}=\sqrt{\frac{2 k-a}{a}}$. To show $D_{1}<C_{1}$ is equal to prove that $\frac{\sqrt{2 k} \sqrt{\frac{1}{a}} \theta_{a, b}}{\left(1-\delta_{a}-\sqrt{\frac{s}{a}} \theta_{a, b}\right) \sqrt{s}}+\frac{1}{\sqrt{k}}<\frac{\sqrt{2 k} \sqrt{\frac{1}{a}} \theta_{a, b}}{\left(1-\delta_{a}-\sqrt{\frac{2 k-a}{a}} \theta_{a, b}\right) \sqrt{2 k-a}}+\frac{1}{\sqrt{k}}$. It suffices to prove $1-\delta_{a}<\frac{\sqrt{2 k-a}+\sqrt{s}}{\sqrt{a}} \theta_{a, b}$, i.e., $1-\delta_{a}-C_{a, b, k}^{\alpha, \omega} \theta_{a, b}<\sqrt{\frac{2 k-a}{a}} \theta_{a, b}$.

\section{Acknowledgments}

This work was supported by the NSF of China (Nos.11271050, 11371183) and Beijing Center for Mathematics and Information Interdisciplinary Sciences (BCMIIS).

\section{References}

[1] R. V. Borries, C. Miosso and C. Potes, Compressed sensing using prior information, in 2nd IEEE Int. Workshop on Computational Advances in Multi-Sensor Adaptive Processing, CAMPSAP 2077, 12-14, 2007, pp. 121-124.

[2] T. T. Cai, L. Wang and G. W. Xu, Shifting inequality and recovery of sparse signals, IEEE Trans. Signal Process, 58(3), pp. 1300-1308, 2010.

[3] T. T. Cai, L. Wang and G. W. Xu, New bounds for restricted isometry constants, IEEE Trans. Inform. Theory, 56(9), pp. 4388-4394, 2010.

[4] T. T. Cai, L. Wang and G. Xu, Stable recovery of sparse signals and an oracle inequality, IEEE, Trans. Inform. Theory, 56(7), pp. 3516-3522, 2010.

[5] T. T. Cai, G. W. Xu, J. Zhang, On recovery of sparse signal via $l_{1}$ minimization, IEEE Trans. Inf. Theory, 55(7), pp. 3388-3397, 2009.

[6] T. T. Cai and A. Zhang, Spares representation of a polytope and recovery of sparse signals and low-rank matrices, IEEE Trans. Inform. Theory, 60(1), pp. 122-132, 2014.

[7] T. T. Cai and A. Zhang, Compressed sensing and affine rank minimization under restricted isometry, IEEE Trans. Signal Process., 61(13), pp. 3279-3290, 2013.

[8] T. T. Cai and A. Zhang, Sharp RIP bound for sparse signal and low-rank matrix recovery, Appl. Comput. Harmon. Anal., 35, pp. 74-93, 2013.

[9] E. J. Candè, J. Romberg and T. Tao, Stable signal recovery from incomplete and inaccurate measurements, Commum. Pure Appl. Math., 59, pp. 1207-1223, 2006. 
[10] E. J. Candè, J. Romberg and T. Tao, Robust uncertainty principles: Exact signal reconstruction from highly incomplete frequency information, IEEE Trans. Inform. Theory, 52(20), pp. 489-509, 2006.

[11] E. J. Candès and T. Tao, The Dantzing selector: Statistical estimation when $p$ is much larger than $n$ (with discussion), Ann. Statist., 35, pp. 2313-2351, 2007.

[12] E. J. Candès and T. Tao, Decoding by linear programming, IEEE Trans. Inf. Theory, 51(12), pp. 4203-4215, 2005.

[13] D. Donoho, Compressed sensing, IEEE Trans. Inform. Theory, 52, pp. 11289-1306, 2006.

[14] D. Donoho and M. Elad, Optimally sparse representation in general (nonorthogonal) dictionaries via $l_{1}$ minimization, Proc. Natl. Acad. Sci. USA, 100(5), pp. 2197-2202, 2003.

[15] D. Donoho and X. Huo, Uncertainty principles and ideal atomic decomposition, IEEE, Trans. Inform. Theory, 47(7), pp. 2845-2862, 2001.

[16] M. P. Friedlander, H. Mansour, R. Saab and O. Yilmaz, Recoverying compressively sampled signals using partial support information, IEEE Transactions Information Theory, 58(2), pp. 1122-1134, 2012.

[17] J. J. Fuchs, On sparse representations in arbitrary redundant bases, IEEE, Trans. Inform. Theory, 50(6), pp.1341-1344, 2004.

[18] L. Jacques, A short note compressed sensing with partially known signal support, Signal Process., 90, pp. 3308-3312, 2010.

[19] M. A. Khajehnejad, W. Xu, A. S. Avestimehr and B. Hassibi, Weighted $l_{1}$ minimization for sparse recovery with prior information, in IEEE Int. Symp. Information Theory, ISIT 2009, 2009, pp. 483-487.

[20] S. Li and H. M. Wang, Signal recovery with partially known signal support (in Chinese), Sci. Sin. Math., 42, pp. 313-319, 2012.

[21] W. Lu and N. Vaswani, Exact reconstruction conditions and error bounds for regularized modified basis pursuit, in Proc. Asilomar Conf. on Signals, Systems and Computers, 2010.

[22] W. Lu and N. Vaswani, Modified basis pursuit denoising (modifiedbpdn) for noisy compressive sensing with partially known signal support, in IEEE Int. Conf. Acoustics Speech and Signal Processing (ICASSP), 2010, 14-19, 2010, pp. 3926-3929. 
[23] Q. Mo and S. Li, New bounds on the restricted isometry constant $\delta_{2 k}$, Appl. Comput. Harmon. Anal., 31(3), pp. 3335460-468, 2011.

[24] N. Vaswani and W. Lu, Modified-CS: Modifying compressive sensing for problems with partially known support, IEEE Trans. Signal Process., 58(9), pp. 4595-4607, 2010.

[25] N. Vaswani and W. Lu, Modified-CS: Modifying compressive sensing for problems with partially known support, IEEE Int. Symp. Information Theory, ISIT 2009, pp. 488-492, 2009.

[26] Z. Q. Xu, Compressd sensing: A survey (in Chinese), Sci. Sin. Math., 42, pp. 865-877, 2012. 